\title{
Subelliptic equations with singular nonlinearities on the Heisenberg group
}

\section{Xinjing Wang and Yongzhong Wang ${ }^{*}$ (D)}

${ }^{\text {"Correspondence: }}$

wangyz@nwpu.edu.cn

Department of Applied

Mathematics, Northwestern

Polytechnical University, Xi'an,

Shaanxi 710129, P.R. China

\section{Abstract}

In this paper, we consider the Dirichlet boundary value problem to singular semilinear subelliptic equation on the Heisenberg group

$$
-\Delta_{\mathbb{H}} u=\frac{1}{u^{\gamma}}+f(u), \quad \gamma>0 .
$$

We prove the positivity and continuity up to the boundary for the weak solutions. We also conclude monotonicity of cylindrical solutions to the problem based on a study of the equation $-\Delta_{\mathbb{H}} u_{0}=\frac{1}{u_{0}^{\gamma}}$. The main technique is a generalization of the moving plane method to the Heisenberg group.

MSC: Primary 35A05; secondary 35J55

Keywords: Heisenberg group; subelliptic equation; singular nonlinearity; monotonicity; moving plane method

\section{Introduction}

Crandall, Rabinowitz, and Tartar [1] considered the Dirichlet problem to elliptic equation with singular nonlinearity and deduced the existence and continuity of solutions. The Dirichlet problem on the sufficiently regular bounded domain to the elliptic equation

$$
\Delta u+p(x) \frac{1}{u^{\gamma}}=0, \quad \gamma>0
$$

was treated by Lazer and Mckenna [2], and the existence of solutions with smoothing in the domain and continuity up to the boundary was given under the assumption $p(x)>0$. Boccardo and Orsina [3] derived the existence, regularity, and nonexistence for the Dirichlet problem to the model

$$
-\Delta u=\frac{f(x)}{u^{\gamma}}
$$

where the results depend on $\gamma$ and the summability of $f(x)$ in some Lebesgue spaces.

Canino and Degiovanni [4] applied a variational approach to the singular semilinear elliptic equation

$$
-\Delta u=u^{-\beta}+g(x, u), \quad \beta>0,
$$

(c) The Author(s) 2018. This article is distributed under the terms of the Creative Commons Attribution 4.0 International License (http://creativecommons.org/licenses/by/4.0/), which permits unrestricted use, distribution, and reproduction in any medium, provided you give appropriate credit to the original author(s) and the source, provide a link to the Creative Commons license, and indicate if changes were made. 
and obtained some properties of solutions $u$ and $u_{0}$, where $u_{0}$ is the solution of the Dirichlet problem to the equation

$$
-\Delta u_{0}=u_{0}^{-\beta}
$$

Based on the approach in [4] and the moving plane method, the monotonicity properties of weak solutions of the Dirichlet problem to the singular elliptic equation

$$
-\Delta u=\frac{1}{u^{\gamma}}+f(u)
$$

were proved by Canino, Grandinetti, and Sciunzi [5].

Along with the development of theory of degenerate elliptic partial differential equations, there were many attempts to extend various results of elliptic equations to subelliptic equations. Birindelli and Prajapat [6] derived a Liouville theorem for the nonnegative cylindrical solutions of the equation

$$
\Delta_{\mathbb{H}} u+u^{p}=0
$$

in the Heisenberg group $\mathbb{H}^{n}=\left(\mathbb{R}^{2 n+1}, \circ\right)$, where $\Delta_{\mathbb{H}}$ is the sub-Laplacian on $\mathbb{H}^{n}, 0<p<\frac{Q+2}{Q-2}$, by using the $\mathrm{CR}$ inversion to a regular function $u$. Xu [7] determined that there exist $C^{\infty}(\bar{\Omega})$ solutions of the Dirichlet problem

$$
\begin{cases}\sum_{j=1}^{m} X_{j}^{*} X_{j}+c u=f(x, u) & \text { in } \Omega \\ u=\varphi & \text { on } \partial \Omega\end{cases}
$$

where $f$ and $\varphi$ satisfy proper smooth conditions, $\left\{X_{j}\right\}(j=1, \ldots, m)$ is a system of vector fields satisfying Hörmander's condition, $\Omega$ is a bounded domain, and its boundary $\partial \Omega$ is smooth and noncharacteristic for $\left\{X_{j}\right\}$. By extending the Perron method in the Euclidean space to the Carnot group and constructing barrier functions, the existence and uniqueness of solutions for the Dirichlet problem

$$
\begin{cases}L u+f(x, u)=0 & \text { in } \Omega, \\ u=\varphi & \text { on } \partial \Omega\end{cases}
$$

where $L$ is the sub-Laplacian on the Carnot group, were established by Yuan and Yuan [8]. We also refer the readers to [9] and [10] for the related results.

The purpose of this paper is to consider the Dirichlet problem in $\mathbb{H}^{n}$ to the subelliptic equation with singular nonlinearities

$$
\begin{cases}-\Delta_{\mathbb{H}} u=\frac{1}{u^{\gamma}}+f(u), & \xi \in \Omega, \\ u=0, & \xi \in \partial \Omega,\end{cases}
$$

where $\Omega$ is a bounded convex domain (see $[11,12]$ ) in $\mathbb{H}^{n}$ with boundary $\partial \Omega$ satisfying the Wiener criterion (for any $a \in(0,1)$, let the $C_{j}$ be the capacity of the set $B_{\mathbb{H}}\left(\xi_{0}, a^{j}\right) \cap \Omega^{C}$; then $\xi_{0} \in \partial \Omega$ is a regular point if and only if $\sum_{j=0}^{\infty} C_{j} / a^{j(Q-2)}$ diverges; see [13, 14] etc.), $\gamma>0$, and $f$ satisfies the following condition: 
(F) $f$ is a nonnegative nondecreasing locally Lipschitz function, $f(s)>0$ for any $s>0$, and $f(0) \geq 0$.

We say that $u \in H^{1}(\Omega)$ is a weak solution to (1.1) if for any $\varphi \in C_{0}^{\infty}(\Omega)$,

$$
\int_{\Omega} \nabla_{\mathbb{H}} u \cdot \nabla_{\mathbb{H}} \varphi=\int_{\Omega}\left(\frac{1}{u^{\gamma}}+f\right) \varphi
$$

We first consider the Dirichlet problem

$$
\begin{cases}-\Delta_{\mathbb{H}} u_{0}=\frac{1}{u_{0} \gamma}, & \xi \in \Omega \\ u_{0}=0, & \xi \in \partial \Omega\end{cases}
$$

By borrowing the method in [3] to our setting we prove that existence, uniqueness, and boundedness of weak positive solutions $u_{n}$ for the approximation problem

$$
\begin{cases}-\Delta_{\mathbb{H}} u_{n}=\frac{1}{\left(u_{n}+\frac{1}{n}\right)^{2}}, & \xi \in \Omega, \\ u_{n}=0, & \xi \in \partial \Omega,\end{cases}
$$

for each fixed $n$ (the bound of $u_{n}$ in $H_{0}^{1}(\Omega)$ is local when $\gamma>1$ ), and then we obtain the existence and uniqueness of a weak solution $u$ of $(1.2)$ as the limit of an increasing sequence of positive solutions $\left\{u_{n}\right\}$. Since $\partial \Omega$ satisfies the Wiener criterion, we use the sub-super solution method similarly to [4] and get that the positive solution $u_{0}$ of (1.2) is continuous up to $\partial \Omega$. The monotonicity of $u_{0}$ is shown via the moving plane method.

Similarly to the method of energy functional in [4], we define the functional $F_{f}: L^{2}(\Omega) \rightarrow$ $(-\infty,+\infty]$ to problem $(1.1)$ by

$$
F_{f}(u)= \begin{cases}\frac{1}{2} \int_{\Omega}\left|\nabla_{\mathbb{H}}\left(u-u_{0}\right)\right|^{2}+\int_{\Omega} G_{0}\left(\xi, u-u_{0}\right)-\int_{\Omega} f\left(\xi, u-u_{0}\right) & \text { if } u \in u_{0}+H_{0}^{1}(\Omega), \\ +\infty & \text { otherwise }\end{cases}
$$

where $G_{0}: \Omega \times \mathbb{R} \rightarrow[0,+\infty]$ is the Borel function

$$
G_{0}(\xi, s)=\Phi\left(u_{0}(\xi)+s\right)-\Phi\left(u_{0}(\xi)\right)+s u_{0}^{-\beta}(\xi)
$$

with

$$
\Phi(s)= \begin{cases}-\int_{1}^{s} t^{-\beta} & \text { if } s \geq 0 \\ +\infty & \text { if } s<0\end{cases}
$$

It follows that $G_{0}(\xi, 0)=0$ and $G_{0}(\xi, \cdot)$ is convex and lower semicontinuous for any $\xi \in \Omega$ and that $F_{f}$ is convex, lower semicontinuous, and coercive with $F_{f}\left(u_{0}\right)=0$. The effective domain of $F_{f}$ is $\left\{u \in L^{2}(\Omega): F_{f}(u)<+\infty\right\}$, which is independent of $f$. Moreover, if $u_{0}$ is the minimum of $F_{0}$, then the effective domain of $F_{f}$ is

$$
\left\{u \in u_{0}+H_{0}^{1}(\Omega)\right\} \subseteq H_{\mathrm{loc}}^{1}(\Omega),
$$


and we see that $u \in H_{\mathrm{loc}}^{1}(\Omega)$ is a weak solution to (1.1). Motivated by the idea in [5], we use the decomposition

$$
u=u_{0}+w
$$

and demonstrate two maximum principles for $w$ satisfying

$$
-\Delta_{\mathbb{H}} w=-\Delta_{\mathbb{H}} u+\Delta_{\mathbb{H}} u_{0}
$$

Then the monotonicity of $w$ is derived. Based on this, we arrive at the monotonicity of $u$.

To obtain the monotonicity of $u$, we apply the moving plane method on $\mathbb{H}^{n}$ in [6] (see also [15-18]) by adopting the simpler way of [15] in the proof. Since $\Delta_{\mathbb{H}}$ is not invariant with respect to the general reflection, Birindelli and Prajapat [6] introduced the so-called Hreflection and pointed out that $\Delta_{\mathbb{H}}$ is invariant with respect to the H-reflection. Applying the H-reflection and the moving plane method, we prove that the cylindrical solution of (1.1) is monotone.

Our main result is the following:

Theorem 1.1 Let $\Omega$ be a bounded convex domain symmetric with respect to $T_{\lambda_{1}}$ about $H$-reflection in $\mathbb{H}^{n}$ such that $\partial \Omega$ satisfies the Wiener criterion. If $u \in H_{0}^{1}(\Omega)(\gamma \leq 1)$ or $u \in$ $H_{\text {loc }}^{1}(\Omega)(\gamma>1)$ is a weak solution to (1.1), then $u$ is positive, and $u \in C(\bar{\Omega})$. If $u$ is a positive cylindrical solution to (1.1), then for any $\lambda_{0}<\lambda<\lambda_{1}$ (see Section 2 ),

$$
u(\xi)<u_{\lambda}(\xi) \quad \text { for any } \xi \in \Sigma_{\lambda}
$$

with respect to $t$ (the definitions of $T_{\lambda_{1}}, u_{\lambda}(\xi)$, and $\Sigma_{\lambda}$ will be given in Section 2).

Since $\Delta_{\mathbb{H}}$ on $\mathbb{H}^{n}$ is essentially different from $\Delta$ on $\mathbb{R}^{n}$, the proof of Theorem 1.1 is also essentially different from those for $\Delta$ on $\mathbb{R}^{n}$. Unlike the Euclidean space case, there is no Poincaré's inequality for the narrow region in $\mathbb{H}^{n}$, so we utilize the fact that the volume of the narrow region is small enough to prove the maximum principle on the narrow region. Because the invariance of $\Delta_{\mathbb{H}}$ does not hold for the reflection $u_{\lambda}(x, y, t)=u(y, 2 \lambda-x, t)$ or $u_{\lambda}(x, y, t)=u(2 \lambda-y, x, t)$, the monotonicity of solutions to $x$ or $y$ cannot be established. The assumption that the boundary satisfies the Wiener criterion is weaker than the outer sphere or cone condition.

The paper is organized as follows. In Section 2, we collect some well-known facts about $\mathbb{H}^{n}$ and the subelliptic operator $\Delta_{\mathbb{H}}$. In Section 3, we give the existence, regularity, and monotonicity of $u_{n}$ to (1.3). Section 4 is devoted to the regularity and monotonicity of $u_{0}$ to (1.2) and to the interior gradient estimate for $u_{0} \in C^{3}(\Omega)$. Finally, in Section 5 , we derive the maximum principles of $w$ and prove Theorem 1.1.

\section{Preliminaries}

In this section, we list some facts related to the Heisenberg group and sub-Laplacian $\Delta_{\mathbb{H}}$. For proofs and more details, we refer, for example, to [19-22]. 
The Heisenberg group $\mathbb{H}^{n}$ is the Euclidean space $\mathbb{R}^{2 n+1}(n \geq 1)$ endowed with the group action o defined by

$$
\xi_{0} \circ \xi=\left(x+x_{0}, y+y_{0}, t+t_{0}+2 \sum_{i=1}^{n}\left(x_{i} y_{i_{0}}-y_{i} x_{i_{0}}\right)\right),
$$

where $\xi=\left(x_{1}, \ldots, x_{n}, y_{1}, \ldots, y_{n}, t\right):=(x, y, t) \in \mathbb{R}^{n} \times \mathbb{R}^{n} \times \mathbb{R}, \xi_{0}=\left(x_{0}, y_{0}, t_{0}\right)$. We denote by $\delta_{\lambda}$ the dilations on $\mathbb{R}^{2 n+1}$, that is,

$$
\delta_{\lambda}(\xi)=\left(\lambda x, \lambda y, \lambda^{2} t\right)
$$

which satisfies $\delta_{\lambda}\left(\xi_{0} \circ \xi\right)=\delta_{\lambda}\left(\xi_{0}\right) \circ \delta_{\lambda}(\xi)$.

The left-invariant vector fields corresponding to $\mathbb{H}^{n}$ are of the form

$$
\begin{aligned}
X_{i} & =\frac{\partial}{\partial x_{i}}+2 y_{i} \frac{\partial}{\partial t}, \quad i=1, \ldots, n, \\
Y_{i} & =\frac{\partial}{\partial y_{i}}-2 x_{i} \frac{\partial}{\partial t}, \quad i=1, \ldots, n, \\
T & =\frac{\partial}{\partial t} .
\end{aligned}
$$

The Heisenberg gradient of a function $u$ is defined as

$$
\nabla_{\mathbb{H}} u=\left(X_{1} u, \ldots, X_{n} u, Y_{1} u, \ldots, Y_{n} u\right)
$$

The sub-Laplacian $\Delta_{\mathbb{H}}$ on $\mathbb{H}^{n}$ is

$$
\Delta_{\mathbb{H}}=\sum_{i=1}^{n} X_{i}^{2}+Y_{i}^{2}
$$

with the expansion

$$
\Delta_{\mathbb{H}}=\sum_{i=1}^{n} \frac{\partial^{2}}{\partial x_{i}^{2}}+\frac{\partial^{2}}{\partial y_{i}^{2}}+4 y_{i} \frac{\partial^{2}}{\partial x_{i} \partial t}-4 x_{i} \frac{\partial^{2}}{\partial y_{i} \partial t}+4\left(x_{i}^{2}+y_{i}^{2}\right) \frac{\partial^{2}}{\partial t^{2}} .
$$

It is easy to check that

$$
\left[X_{i}, Y_{j}\right]=-4 T \delta_{i j}, \quad\left[X_{i}, X_{j}\right]=\left[Y_{i}, Y_{j}\right]=0, \quad i, j=1, \ldots, n,
$$

and $\left\{X_{1}, \ldots, X_{n}, Y_{1}, \ldots, Y_{n}\right\}$ satisfies Hörmander's rank condition (see [22]). In particular, this implies that $\Delta_{\mathbb{H}}$ is hypoelliptic (see [22]) and the solution of equation including $\Delta_{\mathbb{H}}$ satisfies the maximum principle (see [20, 23]).

Denote by $Q=2 n+2$ the homogeneous dimension of $\mathbb{H}^{n}$. The norm $|\xi|_{\mathbb{H}}$ is the distance of $\xi$ to the origin (see [21]):

$$
|\xi|_{\mathbb{H}}=\left(\sum_{i=1}^{n}\left(x_{i}^{2}+y_{i}^{2}\right)^{2}+t^{2}\right)^{\frac{1}{4}}
$$


Afterward, we also denote $r=\left(|x|^{2}+|y|^{2}\right)^{\frac{1}{2}}$. Using this norm, we can define the distance between two points in $\mathbb{H}^{n}$ in the natural way:

$$
d_{\mathbb{H}}(\xi, \eta)=\left|\eta^{-1} \circ \xi\right|_{\mathbb{H}},
$$

where $\eta^{-1}$ denotes the inverse of $\eta$ with respect to the group action $\circ$, that is, $\eta^{-1}=-\eta$.

The open ball of radius $R>0$ centered at $\xi_{0}$ is the set

$$
B_{\mathbb{H}}\left(\xi_{0}, R\right)=\left\{\eta \in \mathbb{H}^{n} \mid d_{\mathbb{H}}\left(\eta, \xi_{0}\right)<R\right\} .
$$

By the dilation of the group, $\xi \rightarrow|\xi|_{\mathbb{H}}$ is homogeneous of degree one with respect to $\delta_{\lambda}$, and

$$
\left|B_{\mathbb{H}}\left(\xi_{0}, R\right)\right|=\left|B_{\mathbb{H}}(0, R)\right|=\left|B_{\mathbb{H}}(0,1)\right| R^{Q},
$$

where $|\cdot|$ denotes the Lebesgue measure. Noting that $X_{i}$ and $Y_{i}$ are homogeneous of degree minus one with respect to $\delta_{\lambda}$, that is,

$$
X_{i}\left(\delta_{\lambda}\right)=\lambda \delta_{\lambda}\left(X_{i}\right), \quad Y_{i}\left(\delta_{\lambda}\right)=\lambda \delta_{\lambda}\left(Y_{i}\right)
$$

we see that $\Delta_{\mathbb{H}}$ is homogeneous of degree minus two and left invariant.

Define the Sobolev space

$$
H^{1}(\Omega)=\left\{u|u,| \nabla_{\mathbb{H}} u \mid \in L^{2}(\Omega)\right\},
$$

which is a Banach space with the norm

$$
\|u\|_{H^{1}(\Omega)}=\|u\|_{L^{2}(\Omega)}+\left\|\nabla_{\mathbb{H}} u\right\|_{L^{2}(\Omega)} .
$$

Denote by $H_{0}^{1}(\Omega)$ the closure of $C_{0}^{\infty}(\Omega)$ in $H^{1}(\Omega)$.

If $u(x, y, t)=u(r, t)$, then we say that $u$ is cylindrical. It is easy to see that

$$
\Delta_{\mathbb{H}} u(r, t)=\frac{\partial^{2} u}{\partial r^{2}}+\frac{2 n-1}{r} \frac{\partial u}{\partial r}+4 r^{2} \frac{\partial^{2} u}{\partial t^{2}} .
$$

If the weak solution $u \in H_{0}^{1}(\Omega)$ to (1.1) is cylindrical, then $u$ is said to be the cylindrical solution to (1.1).

For any $\xi=(x, y, t) \in \mathbb{H}^{n}$ and $\lambda \in \mathbb{R}$, we set

$$
T_{\lambda}=\left\{\xi \in \mathbb{H}^{n} \mid t=\lambda\right\}
$$

and

$$
\Sigma_{\lambda}=\{\xi \in \Omega \mid t<\lambda\} .
$$

Let $\xi_{\lambda}=(y, x, 2 \lambda-t)$ be the H-reflection of $\xi$ about $T_{\lambda}$, and let

$$
\Sigma_{\lambda}^{\prime}=\left\{\xi_{\lambda} \in \Omega \mid \xi \in \Sigma_{\lambda}\right\} .
$$


Denote

$$
\lambda_{0}=\min \left\{\lambda: T_{\lambda} \cap \bar{\Omega} \neq \varnothing\right\}
$$

and

$$
\lambda_{1}=\max \left\{\lambda: \Sigma_{\lambda} \cup T_{\lambda} \cup \Sigma_{\lambda}^{\prime}=\Omega\right\} .
$$

Similarly to [6], we denote by $u_{\lambda}$ the H-reflection of $u$ about $T_{\lambda}$ :

$$
u_{\lambda}(x, y, t)=u_{\lambda}(r, t)=u(r, 2 \lambda-t)=u(y, x, 2 \lambda-t) .
$$

The following result is from [24].

Lemma 2.1 If $\in L^{\infty}(\Omega)$ and $u \in H_{0}^{1}(\Omega)$ is a solution to the subelliptic equation

$$
-\Delta_{\mathbb{H}} u=f,
$$

then

$$
\sup _{\Omega} u \leq C\|f\|_{L^{\infty}(\Omega)}
$$

where $C$ is a positive constant depending only on $n$ and $\Omega$.

Let us state Sobolev's and Poincaré's inequalities in $\mathbb{H}^{n}$; see $[25,26]$.

Lemma 2.2 Let $U$ be a bounded domain in $\mathbb{H}^{n}$, and let $\Omega \Subset U$. If $1<p<Q$ and $u \in$ $W_{0}^{1, p}(\Omega)$, then there exists $C>0$ depending on $n, p$, and $\Omega$, such that for any $1 \leq q \leq \frac{p Q}{Q-p}$,

$$
\left(\int_{\Omega}|u|^{q}\right)^{\frac{1}{q}} \leq C\left(\int_{\Omega}\left|\nabla_{\mathbb{H}} u\right|^{p}\right)^{\frac{1}{p}} .
$$

If $1 \leq p<\infty$ and $u \in W_{0}^{1, p}(\Omega)$, then

$$
\int_{\Omega}|u|^{p} \leq C \int_{\Omega}\left|\nabla_{\mathbb{H}} u\right|^{p}
$$

Lemma 2.3 ([20, 23]) Let $\Omega$ be a bounded domain, and let $K(\xi)>0$. If $u$ satisfies

$$
-\Delta_{\mathbb{H}} u+K u \geq 0 \quad \text { on } \Omega ; \quad u=0 \quad \text { in } \partial \Omega,
$$

then $u \geq 0$ on $\Omega$. Furthermore, $u>0$ on $\Omega$ unless $u \equiv 0$.

\section{Solutions $u_{n}$ to (1.3)}

Let $\Omega$ be a bounded domain of $\mathbb{H}^{n}$, and let $\gamma>0$. In this section, we prove the existence, uniqueness, and boundedness of solutions to the Dirichlet problem (1.3). We will work by approximation similarly to [3]. 
Lemma 3.1 Problem (1.3) has a nonnegative solution $u_{n} \in H_{0}^{1}(\Omega) \cap L^{\infty}(\Omega)$ for any given $n$.

Proof Let $v \in L^{2}(\Omega)$. It is well known that the Dirichlet problem to the subelliptic equation

$$
\begin{cases}-\Delta_{\mathbb{H}} v^{*}=\frac{1}{\left(|v|+\frac{1}{n}\right)^{\gamma}}, & \xi \in \Omega, \\ v^{*}=0, & \xi \in \partial \Omega,\end{cases}
$$

has a unique solution $v^{*}=S(v)$. Multiplying by $v^{*}$ the equation and integrating over $\Omega$, we have

$$
\int_{\Omega}\left|\nabla_{\mathbb{H}} v^{*}\right|^{2}=\int_{\Omega}\left(-\Delta_{\mathbb{H}} v^{*}\right) v^{*}=\int_{\Omega} \frac{v^{*}}{\left(|v|+\frac{1}{n}\right)^{\gamma}} \leq n^{\gamma} \int_{\Omega}\left|v^{*}\right| .
$$

By (2.8) in Lemma 2.2 and Hölder's inequality we obtain

$$
\int_{\Omega}\left(v^{*}\right)^{2} \leq C \int_{\Omega}\left|\nabla_{\mathbb{H}} v^{*}\right|^{2} \leq C n^{\gamma} \int_{\Omega}\left|v^{*}\right| \leq C n^{\gamma}\left(\int_{\Omega}\left|v^{*}\right|^{2}\right)^{\frac{1}{2}}
$$

and

$$
\left\|v^{*}\right\|_{L^{2}(\Omega)} \leq C n^{\gamma}
$$

Then the ball in $L^{2}(\Omega)$ of radius $C n^{\gamma}$ is invariant for $S(v)=|v|$. Using the Sobolev embedding [25], we see that $S$ is continuous and compact on $L^{2}(\Omega)$, and by Schauder's fixed point theorem there exists $u_{n} \in H_{0}^{1}(\Omega)$ such that $u_{n}=S\left(u_{n}\right)$, that is, $u_{n}$ is a solution to the problem

$$
\begin{cases}-\Delta_{\mathbb{H}} u_{n}=\frac{1}{\left(\left|u_{n}\right|+\frac{1}{n}\right)^{\gamma}}, & \xi \in \Omega, \\ u_{n}=0, & \xi \in \partial \Omega .\end{cases}
$$

Noting that $\frac{1}{\left(\left|u_{n}\right|+\frac{1}{n}\right)^{\gamma}} \geq 0$, Lemma 2.3 gives $u_{n} \geq 0$, and then $u_{n}$ solves (1.3). Since $\frac{1}{\left(u_{n}+\frac{1}{n}\right)^{\gamma}}$ in (1.3) belongs to $L^{\infty}(\Omega)$, Lemma 2.1 implies that $u_{n} \in L^{\infty}(\Omega)$.

Lemma 3.2 A solution of (1.3) is unique.

Proof Since $v_{n}$ satisfies

$$
-\Delta_{\mathbb{H}} v_{n}=\frac{1}{\left(v_{n}+\frac{1}{n}\right)^{\gamma}}
$$

in the distribution sense, it follows that

$$
-\Delta_{\mathbb{H}}\left(u_{n}-v_{n}\right)=\frac{1}{\left(u_{n}+\frac{1}{n}\right)^{\gamma}}-\frac{1}{\left(v_{n}+\frac{1}{n}\right)^{\gamma}}=\frac{\left(v_{n}+\frac{1}{n}\right)^{\gamma}-\left(u_{n}+\frac{1}{n}\right)^{\gamma}}{\left(u_{n}+\frac{1}{n}\right)^{\gamma}\left(v_{n}+\frac{1}{n}\right)^{\gamma}} .
$$

Taking $\left(u_{n}-v_{n}\right)^{+}$as the test function and observing that

$$
\left(\left(v_{n}+\frac{1}{n}\right)^{\gamma}-\left(u_{n}+\frac{1}{n}\right)^{\gamma}\right)\left(u_{n}-v_{n}\right)^{+} \leq 0
$$


it follows by (3.2) that

$$
0 \leq \int_{\Omega}\left|\nabla_{\mathbb{H}}\left(u_{n}-v_{n}\right)^{+}\right|^{2} \leq 0,
$$

and therefore $\left(u_{n}-v_{n}\right)^{+}=0$ a.e. in $\Omega$, which implies $u_{n} \leq v_{n}$. In the same way, taking $\left(v_{n}-u_{n}\right)^{+}$as the test function, we can prove that $u_{n} \geq v_{n}$. This proves the uniqueness.

Lemma 3.3 The nonnegative solution sequence $\left\{u_{n}\right\}$ from (1.3) is nondecreasing with respect to $n$, and for every $\omega \Subset \Omega$, there exists a constant $c_{\omega}>0$, independent on $n$, such that, for any $\xi \in \omega$,

$$
u_{n}(\xi) \geq c_{\omega} \text { for every } n
$$

Proof For $\gamma>0$, we have that

$$
-\Delta_{\mathbb{H}} u_{n}=\frac{1}{\left(u_{n}+\frac{1}{n}\right)^{\gamma}} \leq \frac{1}{\left(u_{n}+\frac{1}{n+1}\right)^{\gamma}}
$$

in the distribution sense, and then

$$
\begin{aligned}
-\Delta_{\mathbb{H}}\left(u_{n}-u_{n+1}\right) & \leq \frac{1}{\left(u_{n}+\frac{1}{n+1}\right)^{\gamma}}-\frac{1}{\left(u_{n+1}+\frac{1}{n+1}\right)^{\gamma}} \\
& =\frac{\left(u_{n+1}+\frac{1}{n+1}\right)^{\gamma}-\left(u_{n}+\frac{1}{n+1}\right)^{\gamma}}{\left(u_{n}+\frac{1}{n+1}\right)^{\gamma}\left(u_{n+1}+\frac{1}{n+1}\right)^{\gamma}} .
\end{aligned}
$$

Taking $\left(u_{n}-u_{n+1}\right)^{+}$as the test function in (3.5) and using

$$
\left(\left(u_{n+1}+\frac{1}{n+1}\right)^{\gamma}-\left(u_{n}+\frac{1}{n+1}\right)^{\gamma}\right)\left(u_{n}-u_{n+1}\right)^{+} \leq 0,
$$

we have

$$
0 \leq \int_{\Omega}\left|\nabla_{\mathbb{H}}\left(u_{n}-u_{n+1}\right)^{+}\right|^{2} \leq 0,
$$

which implies $\left(u_{n}-u_{n+1}\right)^{+}=0$ a.e. in $\Omega$, that is, $u_{n} \leq u_{n+1}$, so $\left\{u_{n}\right\}$ is nondecreasing.

Noting that $u_{1} \in L^{\infty}(\Omega)$ by Lemma 3.1 and using Lemma 2.1 to the equation $-\Delta_{\mathbb{H}} u_{1}=$ $\frac{1}{\left(u_{1}+1\right)^{\gamma}} \leq 1$, we get that there exists a constant $C>0$, only depending on $\Omega$ and $Q$, such that $\left\|u_{1}\right\|_{L^{\infty}(\Omega)} \leq C$ and

$$
-\Delta_{\mathbb{H}} u_{1}=\frac{1}{\left(u_{1}+1\right)^{\gamma}} \geq \frac{1}{\left(\left\|u_{1}\right\|_{L^{\infty}(\Omega)}+1\right)^{\gamma}} \geq \frac{1}{(C+1)^{\gamma}} .
$$

Lemma 2.3 implies that $u_{1}>0$ in $\Omega$, and then (3.4) also holds for $u_{1}$. By the monotonicity of $u_{n}$, (3.4) holds for $u_{n}$.

Remark 3.1 Using (3.4), it follows that

$$
-\Delta_{\mathbb{H}} u_{n}=\frac{1}{\left(u_{n}+\frac{1}{n}\right)^{\gamma}} \leq \frac{1}{\left(u_{n}\right)^{\gamma}} \leq \frac{1^{\gamma}}{c},
$$


and Lemma 2.1 yields

$$
\sup _{\omega} u_{n} \leq C
$$

Lemma 3.4 Let $u_{n} \in H_{0}^{1}(\Omega)$ be the solution to (1.3). Then $u_{n}$ is bounded if $0<\gamma \leq 1$ and locally bounded if $\gamma>1$.

Proof If $\gamma>1$, then by taking $u_{n}$ as the test function in (1.3) and using Lemma 3.3 we have

$$
\int_{\Omega}\left|\nabla_{\mathbb{H}} u_{n}\right|^{2}=\int_{\Omega}\left(-\Delta_{\mathbb{H}} u_{n}\right) u_{n}=\int_{\Omega} \frac{u_{n}}{\left(u_{n}+\frac{1}{n}\right)^{\gamma}} \leq \int_{\Omega} \frac{1}{\left(u_{n}+\frac{1}{n}\right)^{\gamma-1}} \leq \frac{|\Omega|}{c_{\omega}^{\gamma-1}} .
$$

This shows that $u_{n}$ is locally bounded. For $0<\gamma \leq 1$, choosing $u_{n}$ as the test function in (1.3) and using Hölder's inequality, we get that

$$
\begin{aligned}
\int_{\Omega}\left|\nabla_{\mathbb{H}} u_{n}\right|^{2} & =\int_{\Omega}\left(-\Delta_{\mathbb{H}} u_{n}\right) u_{n}=\int_{\Omega} \frac{u_{n}}{\left(u_{n}+\frac{1}{n}\right)^{\gamma}} \leq \int_{\Omega} u_{n}^{1-\gamma} \\
& \leq|\Omega|^{1-\frac{1}{m}}\left(\int_{\Omega} u_{n}^{(1-\gamma) m}\right)^{\frac{1}{m}}, \quad m>1 .
\end{aligned}
$$

If $\gamma=1$, then

$$
\int_{\Omega}\left|\nabla_{\mathbb{H}} u_{n}\right|^{2}=\int_{\Omega}\left(-\Delta_{\mathbb{H}} u_{n}\right) u_{n}=\int_{\Omega} \frac{u_{n}}{u_{n}+\frac{1}{n}} \leq|\Omega|
$$

If $0<\gamma<1$, the taking $m$ in (3.7) satisfying $2^{*}=(1-\gamma) m$ and applying (2.7), we arrive at

$$
\left(\int_{\Omega} u_{n}^{2^{*}}\right)^{\frac{1}{2^{*}}} \leq C\left(\int_{\Omega}\left|\nabla_{\mathbb{H}} u_{n}\right|^{2}\right)^{\frac{1}{2}} \leq C|\Omega|^{1-\frac{1}{m}}\left(\int_{\Omega} u_{n}^{2^{*}}\right)^{\frac{1}{2 m}} .
$$

Since $\frac{1}{2^{*}}>\frac{1}{2 m}$, this inequality implies $u_{n} \in L^{2^{*}}(\Omega)$. Using this estimate again together with (3.7), we have

$$
\left\|\nabla_{\mathbb{H}} u_{n}\right\|_{L^{2}(\Omega)} \leq C,
$$

so that $u_{n}$ is bounded in $H_{0}^{1}(\Omega)$.

Remark 3.2 Note that $u_{n} \in C(\bar{\Omega})$ since the right-hand side in (1.3) is nonsingular.

Lemma 3.5 Let $u_{n} \in C(\bar{\Omega}) \cap H_{0}^{1}(\Omega)(\gamma \leq 1)$ and $u_{n} \in C(\bar{\Omega}) \cap H_{\mathrm{loc}}^{1}(\Omega)(\gamma>1)$ be solutions to (1.3). If $u_{n} \leq\left(u_{n}\right)_{\lambda}$ on $\partial \Omega$, then $u_{n} \leq\left(u_{n}\right)_{\lambda}$ in $\Omega$ with respect to $t$, and then $u_{n}<\left(u_{n}\right)_{\lambda}$ in $\Omega$ unless $u_{n} \equiv\left(u_{n}\right)_{\lambda}$.

Proof Since $u_{n}$ satisfies (1.3) and $\Delta_{\mathbb{H}}$ is invariant under the H-reflection, we have

$$
-\Delta_{\mathbb{H}}\left(u_{n}\right)_{\lambda}=\frac{1}{\left(\left(u_{n}\right)_{\lambda}+\frac{1}{n}\right)^{\gamma}} .
$$


Take positive functions $\phi_{i} \in C_{0}^{\infty}(\Omega), \operatorname{supp} \phi_{i} \subseteq \operatorname{supp}\left(u_{n}-\left(u_{n}\right)_{\lambda}\right)^{+}, i=1,2,3, \ldots$, such that $\phi_{i}$ converges to $\left(u_{n}-\left(u_{n}\right)_{\lambda}\right)^{+}$in $C(\bar{\Omega}) \cap H_{0}^{1}(\Omega)$. Using $\phi_{i}$ as the test function in (3.8), we have by combining (1.3) that

$$
\begin{aligned}
0 & \leq \lim _{i \rightarrow \infty} \int_{\Omega} \nabla_{\mathbb{H}}\left(u_{n}-\left(u_{n}\right)_{\lambda}\right) \cdot \nabla_{\mathbb{H}} \phi_{i} \\
& =\lim _{i \rightarrow \infty} \int_{\Omega}\left(-\Delta_{\mathbb{H}}\left(u_{n}-\left(u_{n}\right)_{\lambda}\right)\right) \phi_{i} \\
& =\lim _{i \rightarrow \infty} \int_{\Omega}\left(\frac{1}{\left(u_{n}+\frac{1}{n}\right)^{\gamma}}-\frac{1}{\left(\left(u_{n}\right)_{\lambda}+\frac{1}{n}\right)^{\gamma}}\right) \phi_{i} \\
& =\int_{\Omega} \frac{\left(\left(\left(u_{n}\right)_{\lambda}+\frac{1}{n}\right)^{\gamma}-\left(u_{n}+\frac{1}{n}\right)^{\gamma}\right)\left(u_{n}-\left(u_{n}\right)_{\lambda}\right)^{+}}{\left(u_{n}+\frac{1}{n}\right)^{\gamma}\left(\left(u_{n}\right)_{\lambda}+\frac{1}{n}\right)^{\gamma}} \\
& \leq 0 .
\end{aligned}
$$

Then $\left(u_{n}-\left(u_{n}\right)_{\lambda}\right)^{+}=0$ a.e. in $\Omega$, which implies by Lemma 2.3 that $u_{n} \leq\left(u_{n}\right)_{\lambda}$ in $\Omega$.

Assume that there exists $\xi_{0} \in \Omega$ such that $u_{n}\left(\xi_{0}\right)=\left(u_{n}\right)_{\lambda}\left(\xi_{0}\right)$ and let $R=R\left(\xi_{0}\right)>0$ be such that $B\left(\xi_{0}, R\right) \Subset \Omega$. We have that

$$
-\Delta_{\mathbb{H}}\left(u_{n}-\left(u_{n}\right)_{\lambda}\right)=\frac{1}{\left(u_{n}+\frac{1}{n}\right)^{\gamma}}-\frac{1}{\left(\left(u_{n}\right)_{\lambda}+\frac{1}{n}\right)^{\gamma}} \quad \text { in } B\left(\xi_{0}, R\right)
$$

in the distribution sense. Since $u_{n}\left(\xi_{0}\right)>0$, we can assume that $u_{n}>0$ in $B\left(\xi_{0}, R\right)$ for small $R$. By Lemma 3.3, $u_{n} \geq c_{B\left(\xi_{0}, R\right)}>0$. Therefore,

$$
\frac{1}{\left(u_{n}+\frac{1}{n}\right)^{\gamma}}-\frac{1}{\left(\left(u_{n}\right)_{\lambda}+\frac{1}{n}\right)^{\gamma}}=c(\zeta)\left(u_{n}-\left(u_{n}\right)_{\lambda}\right)
$$

where $c(\zeta)=\frac{-\gamma}{\zeta^{\gamma+1}}$ with $\zeta$ between $u_{n}+\frac{1}{n}$ and $\left(u_{n}\right)_{\lambda}+\frac{1}{n}$. We see that $c(\zeta)$ is bounded by using $u_{n} \geq c_{B\left(\xi_{0}, R\right)}$. Thus there exists a positive constant $\Lambda \geq|c(\zeta)|$ such that

$$
\frac{1}{\left(u_{n}+\frac{1}{n}\right)^{\gamma}}-\frac{1}{\left(\left(u_{n}\right)_{\lambda}+\frac{1}{n}\right)^{\gamma}}+\Lambda w \geq 0 \quad \text { in } B\left(\xi_{0}, R\right)
$$

Putting this into (3.9), we have

$$
-\Delta_{\mathbb{H}}\left(u_{n}-\left(u_{n}\right)_{\lambda}\right)+\Lambda\left(u_{n}-\left(u_{n}\right)_{\lambda}\right) \geq 0 \quad \text { in } B\left(\xi_{0}, R\right)
$$

By Lemma 2.3 this implies that $u_{n} \equiv\left(u_{n}\right)_{\lambda}$ in $B\left(\xi_{0}, R\right)$. The usual covering argument shows that $u_{n} \equiv\left(u_{n}\right)_{\lambda}$ in $\Omega$.

\section{Solutions to (1.2)}

In this section, we get the regularity of solutions to (1.2) by using results to (1.3). Also, we obtain the monotonicity of cylindrical solutions to (1.2). The gradient estimate and Harnack inequality for classical solutions to (1.2) are provided by calculating the Harnack quantity, which is similar to Li [27] and Ma and Wei [28]. 


\subsection{Regularity and symmetry of solutions to (1.2)}

Lemma 4.1 Problem (1.2) allows a unique nonnegative solution $u \in H_{0}^{1}(\Omega)(\gamma \leq 1)$ or $u \in$ $H_{\mathrm{loc}}^{1}(\Omega)(\gamma>1)$, and (3.4) is true for $u_{0}$, that is, $u_{0}(\xi) \geq c_{\omega}$ for $\xi \in \omega \subset \subset \Omega$.

Proof For every $n$, since the solution $u_{n} \in H_{0}^{1}(\Omega)$ to (1.3) is bounded by Lemma 3.4 and $u_{n}$ converges pointwise to $u_{0}$ in $\Omega$ by Remark 3.1, it follows that $u_{n}$ converges weakly to $u_{0}$ in $H_{0}^{1}(\omega), \omega \Subset \Omega$, that is, for any $\varphi \in C_{0}^{1}(\Omega)$ such that $\operatorname{supp} \varphi=\omega$,

$$
\lim _{n \rightarrow \infty} \int_{\omega} \nabla_{\mathbb{H}} u_{n} \cdot \nabla_{\mathbb{H}} \varphi=\int_{\omega} \nabla_{\mathbb{H}} u_{0} \cdot \nabla_{\mathbb{H}} \varphi
$$

Noting that $u_{n}$ satisfies (3.4), we have that, for any $\varphi \in C_{0}^{1}(\Omega)$,

$$
0 \leq\left|\frac{\varphi}{\left(u_{n}+\frac{1}{n}\right)^{\gamma}}\right| \leq \frac{\|\varphi\|_{L^{\infty}(\Omega)}}{c_{\omega}^{\gamma}}
$$

By Lebesgue's dominated convergence theorem,

$$
\lim _{n \rightarrow \infty} \int_{\Omega} \frac{\varphi}{\left(u_{n}+\frac{1}{n}\right)^{\gamma}}=\int_{\Omega} \frac{\varphi}{u_{0}^{\gamma}} .
$$

This implies by (4.1) and (4.2) that problem (1.2) has a solution $u_{0}$.

Using $u_{n} \geq c_{\omega}$ on $\omega \Subset \Omega$, this yields $u_{0} \geq 0$ in $\Omega$. The uniqueness can be proved as in the proof of Lemma 3.2.

For the equation $\Delta_{\mathbb{H}} z=0$ in $\Omega$, by [29] we have $z \in C^{2}(\Omega)$.

Lemma 4.2 If $\partial \Omega$ satisfies the Wiener criterion and $z_{1} \in H_{0}^{1}(\Omega) \cap C^{2}(\Omega)$ solves

$$
-\Delta_{\mathbb{H}} z_{1}=1 \quad \text { in } \Omega,
$$

then $z_{1} \in C(\bar{\Omega})$ is positive with $\left.z_{1}\right|_{\partial \Omega}=0$.

Proof Let $z_{2}(\xi)=\frac{x_{1}^{2}}{2}$. Then $\Delta_{\mathbb{H}} z_{2}=1$. Since $\partial \Omega$ satisfies the Wiener criterion, it follows by [13] (p. 1158) that there exists $z \in C(\bar{\Omega}) \cap C^{2}(\Omega)$ such that

$$
\Delta_{\mathbb{H}} z=0 \quad \text { in } \Omega \text { and }\left.\quad z\right|_{\partial \Omega}=z_{2} .
$$

We easily see that $z-z_{2} \in H_{0}^{1}(\Omega) \cap C^{2}(\Omega)$ satisfies

$$
-\Delta_{\mathbb{H}}\left(z-z_{2}\right)=1 \quad \text { in } \Omega,
$$

and therefore $z_{1}=z-z_{2} \in C(\bar{\Omega})$ is the required function with $z_{1}>0$ and $\left.z\right|_{\partial \Omega}=0$.

Lemma 4.3 If $\partial \Omega$ satisfies the Wiener criterion, then there exists a solution $u_{0}$ to problem (1.2), and $u_{0} \in C(\bar{\Omega})$. 
Proof Taking $u_{0}^{*}=\left((\gamma+1) z_{1}\right)^{\frac{1}{\gamma+1}}$, we have by Lemma 4.2 that

$$
\begin{aligned}
& \Delta_{\mathbb{H}} u_{0}^{*}+\left(u_{0}^{*}\right)^{-\gamma} \\
& \quad=-\gamma\left((\gamma+1) z_{1}\right)^{\frac{-2 \gamma-1}{\gamma+1}}\left|\nabla_{\mathbb{H}} z_{1}\right|^{2}+\left((\gamma+1) z_{1}\right)^{\frac{-\gamma}{\gamma+1}} \Delta_{\mathbb{H}} z_{1}+\left((\gamma+1) z_{1}\right)^{\frac{-\gamma}{\gamma+1}} \\
& \quad=-\gamma\left((\gamma+1) z_{1}\right)^{\frac{-2 \gamma-1}{\gamma+1}}\left|\nabla_{\mathbb{H}} z_{1}\right|^{2} \leq 0 .
\end{aligned}
$$

Noting that $u_{0}$ is nonnegative, we know by (4.3) that

$$
0 \leq u_{0} \leq u_{0}^{*}=\left((\gamma+1) z_{1}\right)^{\frac{1}{\gamma+1}}
$$

that is, $u_{0}^{*}$ and 0 are the super- and subsolutions of (1.2), respectively. This implies the existence of a solution $u_{0}$ to (1.2). Using $z_{1} \in C(\bar{\Omega})$ in Lemma 4.2, we have $u_{0} \in C(\bar{\Omega})$.

Lemma 4.4 If $\partial \Omega$ satisfies the Wiener criterion, $u_{0} \in C(\bar{\Omega}) \cap H_{0}^{1}(\Omega)(\gamma \leq 1)$ or $u_{0} \in C(\bar{\Omega}) \cap$ $H_{\mathrm{loc}}^{1}(\Omega)(\gamma>1)$ is a cylindrical solution to problem (1.2), then for any $\lambda_{0}<\lambda<\lambda_{1}$, we have

$$
u_{0}(\xi)<\left(u_{0}\right)_{\lambda}(\xi) \text { for all } \xi \in \Sigma_{\lambda}
$$

with respect to $t$.

Proof Step 1 . Suppose that $\lambda$ is sufficiently close to $\lambda_{0}$. Then by Lemma 3.3 we have that $u_{n}>0$ in $\Sigma_{\lambda}$, and then $u_{n} \leq\left(u_{n}\right)_{\lambda}$ on $\partial \Sigma_{\lambda}$. Since $u_{n}=\left(u_{n}\right)_{\lambda}$ on the hyperplane $T_{\lambda}$, it follows that $u_{n} \leq\left(u_{n}\right)_{\lambda}$ in $\Sigma_{\lambda}$, and then $u_{n}<\left(u_{n}\right)_{\lambda}$ on $\Sigma_{\lambda}$ by Lemma 3.5.

Step 2. Since Step 1 provides a starting point, we can move the plane $T_{\lambda}$ to the right keeping $u_{n} \leq\left(u_{n}\right)_{\lambda}$ on $\Sigma_{\lambda}$ until its limiting position $\lambda_{1}$. Denoting

$$
\bar{\lambda}=\sup \left\{\lambda \mid u_{n} \leq\left(u_{n}\right)_{\lambda} \text { in } \Sigma_{\mu}, \mu \leq \lambda\right\},
$$

we claim that $\bar{\lambda}=\lambda_{1}$. Indeed, suppose $\bar{\lambda}<\lambda_{1}$; we will show that the plane $T_{\lambda}$ can be moved further to the right or, to be more rigorous, there exists $\varepsilon>0$ such that, for any $\lambda \in(\bar{\lambda}, \bar{\lambda}+\varepsilon)$,

$$
u_{n} \leq\left(u_{n}\right)_{\lambda}
$$

This is a contradiction to the definition of $\bar{\lambda}$. Hence $\bar{\lambda}=\lambda_{1}$.

To prove (4.5), we separate $\Sigma_{\bar{\lambda}+\varepsilon}$ into two parts, $\Sigma_{\bar{\lambda}-\varepsilon}$ and $\Sigma_{\bar{\lambda}+\varepsilon} \backslash \Sigma_{\bar{\lambda}-\varepsilon}$ for $\varepsilon>0$.

Clearly, $\left(u_{n}\right)_{\bar{\lambda}}-u_{n} \geq c_{\varepsilon}>0$ in $\Sigma_{\bar{\lambda}-\varepsilon}$ for any $\varepsilon>0$. Since $\left(u_{n}\right)_{\lambda}-u_{n}$ depends continuously on $\lambda$, there exists $\varepsilon>0$ sufficiently small such that, for all $\lambda \in(\bar{\lambda}, \bar{\lambda}+\varepsilon)$, we have

$$
\left(u_{n}\right)_{\lambda}-u_{n} \geq 0 \quad \text { in } \Sigma_{\bar{\lambda}-\varepsilon} .
$$

On the other hand, since $\left(u_{n}\right)_{\lambda}-u_{n} \geq 0$ on $\partial\left(\Sigma_{\bar{\lambda}+\varepsilon} \backslash \Sigma_{\bar{\lambda}-\varepsilon}\right)$ for fixed $\varepsilon>0$, it follows by Lemma 3.5 that

$$
\left(u_{n}\right)_{\lambda}-u_{n} \geq 0 \quad \text { in } \Sigma_{\bar{\lambda}+\varepsilon} \backslash \Sigma_{\bar{\lambda}-\varepsilon} .
$$

Combining (4.6) and (4.7), we prove (4.5). 
Using the equality $\bar{\lambda}=\lambda_{1}$ and the convergence of $u_{n}$ to $u_{0}$ a.e., we get that $u_{0} \leq\left(u_{0}\right)_{\lambda}$ on $\Sigma_{\lambda}$. So (4.4) is proved by Lemma 2.3 .

\subsection{Harnack inequality for a solution to (1.2)}

Results in the subsection are not used in the sequel, but they are of independent interest.

Lemma 4.5 Let $u_{0} \in C^{3}(\Omega)$ be a positive solution to (1.2). Then, for any $R=R\left(\xi_{0}\right)>0$, $\xi_{0} \in \Omega$, and $B\left(\xi_{0}, 2 R\right) \subset \Omega$, there exists a positive constant $C=C(R)$ such that

$$
\left|\nabla_{\mathbb{H}} u_{0}\right|^{2} \leq C u_{0}^{2} \quad \text { in } B\left(\xi_{0}, R\right)
$$

Proof Let $w_{0}=\log u_{0}$. Then

$$
\nabla_{\mathbb{H}} w_{0}=u_{0}^{-1} \nabla_{\mathbb{H}} u_{0}
$$

and

$$
\Delta_{\mathbb{H}} w_{0}=-\left|\nabla_{\mathbb{H}} w_{0}\right|^{2}-\frac{1}{u_{0}^{\gamma+1}} .
$$

Taking a smooth cut-off function $\phi$ with $\phi=1$ in $B\left(\xi_{0}, R\right)$ and $\phi=0$ outside $B\left(\xi_{0}, 2 R\right)$, define

$$
P=\phi\left|\nabla_{\mathbb{H}} w_{0}\right|^{2}
$$

which as usual is called the Harnack quantity.

At the maximum point of $P$, we have $\nabla_{\mathbb{H}} P=0$ and $\Delta_{\mathbb{H}} P \leq 0$. Then

$$
\begin{aligned}
& \nabla_{\mathbb{H}}\left(\left|\nabla_{\mathbb{H}} w_{0}\right|^{2}\right)=-\phi^{-1} \nabla_{\mathbb{H}} \phi\left|\nabla_{\mathbb{H}} w_{0}\right|^{2}=-\phi^{-2} P \nabla_{\mathbb{H}} \phi \\
& \left|\nabla_{\mathbb{H}} w_{0}\right|^{2} \Delta_{\mathbb{H}} \phi+2 \nabla_{\mathbb{H}} \phi \cdot \nabla_{\mathbb{H}}\left(\left|\nabla_{\mathbb{H}} w_{0}\right|^{2}\right)+\phi \Delta_{\mathbb{H}}\left(\left|\nabla_{\mathbb{H}} w_{0}\right|^{2}\right) \leq 0 .
\end{aligned}
$$

Using the basic formula

$$
\Delta_{\mathbb{H}}\left|\nabla_{\mathbb{H}} v\right|^{2}=2\left(\left(\nabla_{\mathbb{H}} \Delta_{\mathbb{H}} v, \nabla_{\mathbb{H}} v\right)+\left|D_{\mathbb{H}}^{2} v\right|^{2}\right)
$$

for any smooth function $v$ in $\mathbb{H}^{n}$, it follows

$$
\phi \Delta_{\mathbb{H}}\left(\left|\nabla_{\mathbb{H}} w_{0}\right|^{2}\right)=2 \phi\left(\nabla_{\mathbb{H}} \Delta_{\mathbb{H}} w_{0}, \nabla_{\mathbb{H}} w_{0}\right)+2 \phi\left|D_{\mathbb{H}}^{2} w_{0}\right|^{2}
$$

Let us estimate the right-hand side in (4.14). For the first term, we have

$$
\begin{aligned}
2 \phi\left(\nabla_{\mathbb{H}} \Delta_{\mathbb{H}} w_{0}, \nabla_{\mathbb{H}} w_{0}\right) & =2 \phi\left(\nabla_{\mathbb{H}}\left(-\left|\nabla_{\mathbb{H}} w_{0}\right|^{2}-\frac{1}{u_{0}^{\gamma+1}}\right), \nabla_{\mathbb{H}} w_{0}\right) \\
& =-2 \phi\left(\nabla_{\mathbb{H}}\left(\left|\nabla_{\mathbb{H}} w_{0}\right|^{2}\right), \nabla_{\mathbb{H}} w_{0}\right)+2(\gamma+1) \phi\left(\frac{\nabla_{\mathbb{H}} u_{0}}{u_{0}^{\gamma+2}}, \nabla_{\mathbb{H}} w_{0}\right) \\
& =-2 \phi\left(-\phi^{-2} P \nabla_{\mathbb{H}} \phi, \nabla_{\mathbb{H}} w_{0}\right)+2(\gamma+1) \phi \frac{\left|\nabla_{\mathbb{H}} w_{0}\right|^{2}}{u_{0}^{\gamma+1}}
\end{aligned}
$$




$$
\begin{aligned}
& =2 \phi^{-1}\left(\nabla_{\mathbb{H}} \phi, \nabla_{\mathbb{H}} w_{0}\right) P+\frac{2(\gamma+1)}{u_{0}^{\gamma+1}} P \\
& \geq-\phi^{-1}\left|\nabla_{\mathbb{H}} \phi\right|^{2} P-\phi^{-1}\left|\nabla_{\mathbb{H}} w_{0}\right|^{2} P+\frac{2(\gamma+1)}{u_{0}^{\gamma+1}} P \\
& =-\phi^{-1}\left|\nabla_{\mathbb{H}} \phi\right|^{2} P-\phi^{-2} P^{2}+\frac{2(\gamma+1)}{u_{0}^{\gamma+1}} P .
\end{aligned}
$$

Due to (4.10), the second term of (4.14) satisfies

$$
\begin{aligned}
2 \phi\left|D_{\mathbb{H}}^{2} w_{0}\right|^{2} & \geq 2 \phi\left|\Delta_{\mathbb{H}} w_{0}\right|^{2} \\
& =\left.2 \phi|-| \nabla_{\mathbb{H}} w_{0}\right|^{2}-\left.\frac{1}{u_{0}^{\gamma+1}}\right|^{2} \\
& =2 \phi\left|\nabla_{\mathbb{H}} w_{0}\right|^{4}+\frac{4 \phi\left|\nabla_{\mathbb{H}} w_{0}\right|^{2}}{u_{0}^{\gamma+1}}+\frac{2 \phi}{u_{0}^{2(\gamma+1)}} \\
& \geq \frac{2}{\phi} P^{2}+\frac{4}{u_{0}^{\gamma+1}} P .
\end{aligned}
$$

Substituting (4.15) and (4.16) into (4.14), we obtain

$$
\phi \Delta_{\mathbb{H}}\left(\left|\nabla_{\mathbb{H}} w_{0}\right|^{2}\right) \geq-\frac{\left|\nabla_{\mathbb{H}} \phi\right|^{2}}{\phi} P-\frac{P^{2}}{\phi^{2}}+\frac{2 \gamma+6}{u_{0}^{\gamma+1}} P+\frac{2}{\phi} P^{2} .
$$

From (4.12), $\left|\nabla_{\mathbb{H}} w_{0}\right|^{2}=\frac{P}{\phi},(4.11)$, and (4.17) we have that

$$
\begin{aligned}
0 & \geq \phi \Delta_{\mathbb{H}}\left(\left|\nabla_{\mathbb{H}} w_{0}\right|^{2}\right)+\frac{\Delta_{\mathbb{H}} \phi}{\phi} P-\frac{2\left|\nabla_{\mathbb{H}} \phi\right|^{2}}{\phi} P \\
& \geq \frac{\Delta_{\mathbb{H}} \phi}{\phi} P-\frac{2\left|\nabla_{\mathbb{H}} \phi\right|^{2}}{\phi^{2}} P-\frac{\left|\nabla_{\mathbb{H}} \phi\right|^{2}}{\phi} P-\frac{P^{2}}{\phi^{2}}+\frac{2 \gamma+6}{u_{0}^{\gamma+1}} P+\frac{2}{\phi} P^{2},
\end{aligned}
$$

and so

$$
-\frac{\Delta_{\mathbb{H}} \phi}{\phi}+\frac{2\left|\nabla_{\mathbb{H}} \phi\right|^{2}}{\phi^{2}}+\frac{\left|\nabla_{\mathbb{H}} \phi\right|^{2}}{\phi}-\frac{2 \gamma+6}{u_{0}^{\gamma+1}} \geq \frac{2 \phi-1}{\phi^{2}} P .
$$

This gives

$$
\frac{2 \phi-1}{\phi^{2}} P \leq-\frac{\Delta_{\mathbb{H}} \phi}{\phi}+\frac{2\left|\nabla_{\mathbb{H}} \phi\right|^{2}}{\phi^{2}}+\frac{\left|\nabla_{\mathbb{H}} \phi\right|^{2}}{\phi} \quad \text { on } B\left(\xi_{0}, 2 R\right) .
$$

Using the bounds $\left|\nabla_{\mathbb{H}} \phi\right| \leq C R^{-1}$ and $\left|\Delta_{\mathbb{H}} \phi\right| \leq C R^{-2}$, we get

$$
(2 \phi-1) P \leq-\phi \Delta_{\mathbb{H}} \phi+2\left|\nabla_{\mathbb{H}} \phi\right|^{2}+\phi\left|\nabla_{\mathbb{H}} \phi\right|^{2} \leq \frac{C}{R^{2}} .
$$

Since $\phi=1$ on $B\left(\xi_{0}, R\right)$, by (4.19) we have that

$$
\left|\nabla_{\mathbb{H}} w_{0}\right|^{2} \leq C(R) \quad \text { on } B\left(\xi_{0}, R\right),
$$

which implies (4.8). 
Theorem 4.6 Let $u_{0} \in C^{3}(\Omega)$ be a positive solution to (1.2). Then, for any $R=R\left(\xi_{0}\right)>0$, $\xi_{0} \in \Omega$, and $B\left(\xi_{0}, 2 R\right) \subset \Omega$, there exists $C=C(R)>0$ such that, for all $\xi, \eta \in B\left(\xi_{0}, \frac{R}{2}\right)$,

$$
u_{0}(\xi) \leq C u_{0}(\eta)
$$

Proof Let $I$ be the shortest curve in $B\left(\xi_{0}, R\right)$ joining $\xi$ to $\eta$. Then the length of $I$ is clearly at most $2 R$. Integrating the quantity $\left|\nabla_{\mathbb{H}} \log u_{0}\right|$ along $I$ yields

$$
\log u_{0}(\xi)-\log u_{0}(\eta) \leq \int_{I}\left|\nabla_{\mathbb{H}} \log u_{0}\right|
$$

Applying (4.20), we get

$$
\int_{I}\left|\nabla_{\mathbb{H}} \log u_{0}\right| \leq \int_{I} C \leq C
$$

Now (4.21) is obtained from (4.22) and (4.23).

\section{Properties of $w$ and proof of Theorem 1.1}

In this section, we prove several properties of $w=u-u_{0}$. Similarly to [5], we provide two maximum principles; see Lemmas 5.2 and 5.4. The idea to check monotonicity is inspired by $[5,15]$.

Lemma 5.1 If $\partial \Omega$ satisfies the Wiener criterion, then the weak solution $u \in H_{0}^{1}(\Omega)(\gamma \leq 1)$ or $u \in H_{\mathrm{loc}}^{1}(\Omega)(\gamma>1)$ to problem $(1.1)$ is positive, and $u \in C(\bar{\Omega})$.

Proof By the hypothesis $f \geq 0$ we sees that $u$ is a supersolution to the equation

$$
-\Delta_{\mathbb{H}} u_{0}=\frac{1}{u_{0}^{\gamma}} .
$$

Therefore, $u \geq u_{0}>0$ in $\Omega$.

Since $u=u_{0}+w$, it follows that $w \geq 0$ in $\Omega$. Due to (1.4), we have that $w$ satisfies

$$
\Delta_{\mathbb{H}} w+\frac{1}{\left(u_{0}+w\right)^{\gamma}}+f\left(u_{0}+w\right)-\frac{1}{u_{0}^{\gamma}}=0 \quad \text { in } \Omega ;\left.\quad w\right|_{\partial \Omega}=0 .
$$

Since the equation in (5.1) has no any singularity with respect to $w$, the continuity in $\Omega$ of $w$ is clearly obtained; refer to $[7,8,10]$ etc. Now we only need to check the continuity of $w$ near the boundary $\partial \Omega$.

Indeed, by the nonnegativity of $f$ we easily get that $w_{*}=0$ is a subsolution to (5.1). Also, note that $w^{*}=\left((\gamma+1) z_{1}\right)^{\frac{1}{\gamma+1}}$ satisfies

$$
\Delta_{\mathbb{H}} w^{*}+f\left(u_{0}+w^{*}\right) \leq 0
$$

that is,

$$
-\gamma\left((\gamma+1) z_{1}\right)^{\frac{-2 \gamma-1}{\gamma+1}}\left|\nabla_{\mathbb{H}} z_{1}\right|^{2}-\left((\gamma+1) z_{1}\right)^{\frac{-\gamma}{\gamma+1}}+f\left(u_{0}+\left((\gamma+1) z_{1}\right)^{\frac{1}{\gamma+1}}\right) \leq 0
$$


near the boundary $\left(z_{1} \rightarrow 0\right.$ at this moment), which yields

$$
0 \leq w \leq\left((\gamma+1) z_{1}\right)^{\frac{1}{\gamma+1}}
$$

near the boundary, and this shows that $w$ is continuous near $\partial \Omega$ and then $w \in C(\bar{\Omega})$. Hence $u=u_{0}+w \in C(\bar{\Omega})$.

Lemma 5.2 Suppose that $\partial \Omega$ satisfies the Wiener criterion, $u \in C(\bar{\Omega}) \cap H_{0}^{1}(\Omega)(\gamma \leq 1)$ or $u \in C(\bar{\Omega}) \cap H_{\mathrm{loc}}^{1}(\Omega)(\gamma>1)$ is a solution to problem (1.1), $f$ is locally Lipschitz continuous, $f(s)>0$ for any $s>0$, and $f(0) \geq 0$. Then $u>u_{0}$ in $\Omega$, and $w>0$ in $\Omega$.

Proof By contradiction assume that there exists $\xi_{0} \in \Omega$ such that

$$
w\left(\xi_{0}\right)=u\left(\xi_{0}\right)-u_{0}\left(\xi_{0}\right)=0 .
$$

Take $R=R\left(\xi_{0}\right)>0$ such that $B\left(\xi_{0}, R\right) \subset \subset \Omega$. Then

$$
-\Delta_{\mathbb{H}} w=-\Delta_{\mathbb{H}} u+\Delta_{\mathbb{H}} u_{0}=\frac{1}{\left(u_{0}+w\right)^{\gamma}}+f(u)-\frac{1}{u_{0}^{\gamma}} \geq \frac{1}{\left(u_{0}+w\right)^{\gamma}}-\frac{1}{u_{0}^{\gamma}} \quad \text { in } B\left(\xi_{0}, R\right) .
$$

Since $u_{0}\left(\xi_{0}\right)>0$ by Lemma 4.1 , we can assume that $u_{0}>0$ in $B\left(\xi_{0}, R\right)$. Since

$$
\frac{1}{\left(u_{0}+w\right)^{\gamma}}-\frac{1}{u_{0}^{\gamma}}=c(\xi)\left(u_{0}+w-u_{0}\right)=c(\xi) w
$$

and $|c(\xi)| \leq \Lambda$ for a positive constant $\Lambda$, it follows that

$$
\frac{1}{\left(u_{0}+w\right)^{\gamma}}-\frac{1}{u_{0}^{\gamma}}+\Lambda w \geq 0 \quad \text { in } B\left(\xi_{0}, R\right) .
$$

Combining this with (5.3), we get

$$
-\Delta_{\mathbb{H}} w+\Lambda w \geq 0 \quad \text { in } B\left(\xi_{0}, R\right) .
$$

By Lemma 2.3 we have $w \equiv 0$ in $B\left(\xi_{0}, R\right)$. The covering argument shows that $w \equiv 0$ in $\Omega$. Therefore $f \equiv 0$ by (5.3), which is impossible.

Lemma 5.3 ([5]) For $\gamma>0$, consider the function

$$
g_{\gamma}(a, b, c, d):=a^{\gamma}(a+b)^{\gamma}(c+d)^{\gamma}+a^{\gamma} c^{\gamma}(c+d)^{\gamma}-c^{\gamma}(a+b)^{\gamma}(c+d)^{\gamma}-a^{\gamma} c^{\gamma}(a+b)^{\gamma},
$$

where $(a, b, c, d) \in D:=\{(a, b, c, d) \mid 0 \leq a \leq c ; 0 \leq d \leq b\} \subset \mathbb{R}^{4}$. Then $g_{\gamma} \leq 0$ in $D$.

Lemma 5.4 (Narrow region maximum principle) Assume that $\partial \Omega$ satisfies the Wiener criterion and $T$ is a hyperplane in $\mathbb{H}^{n}$. For $\lambda \in \mathbb{R}$, denote

$$
\begin{aligned}
& T_{\lambda}=\left\{\xi=(x, y, t) \in \mathbb{H}^{n} \mid t=\lambda\right\}, \\
& H=\left\{\xi=(x, y, t) \in \mathbb{H}^{n} \mid t<\lambda\right\} .
\end{aligned}
$$


Let $\Omega^{\prime}$ be a bounded narrow region in $H$ contained in $\left\{\xi \in \mathbb{H}^{n} \mid \lambda-l<t<\lambda\right\}$ with small $l>0$. Suppose that $u \in C(\bar{\Omega}) \cap H_{0}^{1}(\Omega)(\gamma \leq 1)$ or $u \in C(\bar{\Omega}) \cap H_{\mathrm{loc}}^{1}(\Omega)(\gamma>1)$ is a cylindrical solution to problem (1.1) and that $f$ satisfies $(\mathrm{F})$. If $w \leq w_{\lambda}$ on $\partial \Omega^{\prime}$, then for a sufficiently small $l$, we have $w \leq w_{\lambda}$ in $\Omega^{\prime}$ with respect to . Furthermore, $w<w_{\lambda}$ in $\Omega^{\prime}$, unless $w \equiv w_{\lambda}$.

Proof Since $u$ satisfies problem (1.1), we have in the distribution sense that

$$
-\Delta_{\mathbb{H}}\left(u_{0}+w\right)=\frac{1}{\left(u_{0}+w\right)^{\gamma}}+f\left(u_{0}+w\right) \quad \text { in } \Omega
$$

and

$$
-\Delta_{\mathbb{H}}\left(\left(u_{0}\right)_{\lambda}+w_{\lambda}\right)=\frac{1}{\left(\left(u_{0}\right)_{\lambda}+w_{\lambda}\right)^{\gamma}}+f\left(\left(u_{0}\right)_{\lambda}+w_{\lambda}\right) \quad \text { in } \Omega
$$

Also,

$$
-\Delta_{\mathbb{H}}\left(u_{0}\right)_{\lambda}=\frac{1}{\left(u_{0}\right)_{\lambda}^{\gamma}} \quad \text { in } \Omega,
$$

and

$$
\begin{aligned}
-\Delta_{\mathbb{H}} w_{\lambda} & =-\Delta_{\mathbb{H}}\left(\left(u_{0}\right)_{\lambda}+w_{\lambda}\right)+\Delta_{\mathbb{H}}\left(u_{0}\right)_{\lambda} \\
& =\frac{1}{\left(\left(u_{0}\right)_{\lambda}+w_{\lambda}\right)^{\gamma}}+f\left(\left(u_{0}\right)_{\lambda}+w_{\lambda}\right)-\frac{1}{\left(u_{0}\right)_{\lambda}^{\gamma}} \quad \text { in } \Omega .
\end{aligned}
$$

Consider positive functions $\psi_{i} \in C_{0}^{\infty}\left(\Omega^{\prime}\right)$ with $\operatorname{supp} \psi_{i} \subseteq \operatorname{supp}\left(w-w_{\lambda}\right)^{+}$and $\psi_{i} \rightarrow$ $\left(w-w_{\lambda}\right)^{+} \in H_{0}^{1}\left(\Omega^{\prime}\right)$ in $H_{0}^{1}\left(\Omega^{\prime}\right)$. Taking $\psi_{i}$ as the test function, by (5.1) and (5.7) we get that

$$
\begin{aligned}
& \int_{\Omega^{\prime}} \nabla_{\mathbb{H}}\left(w-w_{\lambda}\right) \cdot \nabla_{\mathbb{H}} \psi_{i} \\
& =\int_{\Omega^{\prime}}\left(-\Delta_{\mathbb{H}}\left(w-w_{\lambda}\right)\right) \psi_{i} \\
& =\int_{\Omega^{\prime}}\left(\frac{1}{\left(u_{0}+w\right)^{\gamma}}+f\left(u_{0}+w\right)-\frac{1}{u_{0}^{\gamma}}-\frac{1}{\left(\left(u_{0}\right)_{\lambda}+w_{\lambda}\right)^{\gamma}}-f\left(\left(u_{0}\right)_{\lambda}+w_{\lambda}\right)+\frac{1}{\left(u_{0}\right)_{\lambda}^{\gamma}}\right) \psi_{i} \\
& =\int_{\Omega^{\prime}}\left(\frac{1}{\left(u_{0}\right)_{\lambda}^{\gamma}}-\frac{1}{u_{0}^{\gamma}}+\frac{1}{\left(u_{0}+w\right)^{\gamma}}-\frac{1}{\left(\left(u_{0}\right)_{\lambda}+w_{\lambda}\right)^{\gamma}}\right) \psi_{i} \\
& \quad+\int_{\Omega^{\prime}}\left(f\left(u_{0}+w\right)-f\left(\left(u_{0}\right)_{\lambda}+w_{\lambda}\right)\right) \psi_{i} .
\end{aligned}
$$

Note that $u_{0} \leq\left(u_{0}\right)_{\lambda}$ in $\Omega^{\prime} \cap H$ by Lemma 4.4 and that $w \geq w_{\lambda}$ on supp $\psi_{i}$. Applying Lemma 5.3 with $a=u_{0}, c=\left(u_{0}\right)_{\lambda}, b=w$, and $d=w_{\lambda}$, we have

$$
\frac{1}{\left(u_{0}\right)_{\lambda}^{\gamma}}-\frac{1}{u_{0}^{\gamma}}+\frac{1}{\left(u_{0}+w\right)^{\gamma}}-\frac{1}{\left(\left(u_{0}\right)_{\lambda}+w_{\lambda}\right)^{\gamma}} \leq 0
$$


Since $f$ is locally Lipschitz continuous and nondecreasing, it follows that (5.8) and (5.9) imply

$$
\begin{aligned}
\int_{\Omega^{\prime}} \nabla_{\mathbb{H}}\left(w-w_{\lambda}\right) \cdot \nabla_{\mathbb{H}} \psi_{i} & \leq \int_{\Omega^{\prime}}\left(f\left(u_{0}+w\right)-f\left(\left(u_{0}\right)_{\lambda}+w_{\lambda}\right)\right) \psi_{i} \\
& \leq \int_{\Omega^{\prime}}\left(f\left(\left(u_{0}\right)_{\lambda}+w\right)-f\left(\left(u_{0}\right)_{\lambda}+w_{\lambda}\right)\right) \psi_{i} \\
& \leq C \int_{\Omega^{\prime}}\left(w-w_{\lambda}\right) \psi_{i} .
\end{aligned}
$$

Since $\psi_{i}$ converges to $\left(w-w_{\lambda}\right)^{+}$, by Hölder's inequality and Lemma 2.2 we have

$$
\begin{aligned}
\int_{\Omega^{\prime}}\left|\nabla_{\mathbb{H}}\left(w-w_{\lambda}\right)^{+}\right|^{2} & \leq C \int_{\Omega^{\prime}}\left|\left(w-w_{\lambda}\right)^{+}\right|^{2} \\
& \leq C\left|\Omega^{\prime}\right|^{\frac{Q}{2}}\left(\int_{\Omega^{\prime}}\left|\left(w-w_{\lambda}\right)^{+}\right|^{\frac{2 Q}{Q-2}}\right)^{\frac{Q-2}{Q}} \\
& \leq C\left|\Omega^{\prime}\right|^{\frac{Q}{2}} \int_{\Omega^{\prime}}\left|\nabla_{\mathbb{H}}\left(w-w_{\lambda}\right)^{+}\right|^{2} .
\end{aligned}
$$

Since $C\left|\Omega^{\prime}\right|^{\frac{Q}{2}}<1$ for sufficiently small $l$, it follows that

$$
\int_{\Omega^{\prime}}\left|\nabla_{\mathbb{H}}\left(w-w_{\lambda}\right)^{+}\right|^{2}=0
$$

and then $\left(w-w_{\lambda}\right)^{+}=0$, that is, $w \leq w_{\lambda}$ in $\Omega^{\prime}$.

Assume that there exists $\xi_{0} \in \Omega^{\prime}$ such that $w\left(\xi_{0}\right)=w_{\lambda}\left(\xi_{0}\right)$. We have from (5.1) and (5.7) that

$$
\begin{aligned}
-\Delta_{\mathbb{H}}\left(w_{\lambda}-w\right) & \\
= & \left(\frac{1}{u_{0}^{\gamma}}-\frac{1}{\left(u_{0}\right)_{\lambda}^{\gamma}}+\frac{1}{\left(\left(u_{0}\right)_{\lambda}+w\right)^{\gamma}}-\frac{1}{\left(u_{0}+w\right)^{\gamma}}\right)+\frac{1}{\left(\left(u_{0}\right)_{\lambda}+w_{\lambda}\right)^{\gamma}}-\frac{1}{\left(\left(u_{0}\right)_{\lambda}+w\right)^{\gamma}} \\
& +\left(f\left(\left(u_{0}\right)_{\lambda}+w_{\lambda}\right)-f\left(u_{0}+w\right)\right) .
\end{aligned}
$$

Noting that $u_{0} \leq\left(u_{0}\right)_{\lambda}$ in $\Omega^{\prime}$ by Lemma 4.4 and $w \leq w_{\lambda}$ in $\Omega^{\prime}$ from the previous result,by the monotonicity of $f$ we obtain that

$$
f\left(\left(u_{0}\right)_{\lambda}+w_{\lambda}\right)-f\left(u_{0}+w\right) \geq 0 \text { in } \Omega^{\prime} .
$$

Applying Lemma 5.3 yields

$$
\frac{1}{\left(u_{0}\right)_{\lambda}^{\gamma}}-\frac{1}{u_{0}^{\gamma}}+\frac{1}{\left(u_{0}+w\right)^{\gamma}}-\frac{1}{\left(\left(u_{0}\right)_{\lambda}+w_{\lambda}\right)^{\gamma}} \leq 0 \quad \text { in } \Omega^{\prime} .
$$

Putting (5.14) and (5.15) into (5.13) shows that

$$
-\Delta_{\mathbb{H}}\left(w_{\lambda}-w\right) \geq \frac{1}{\left(\left(u_{0}\right)_{\lambda}+w_{\lambda}\right)^{\gamma}}-\frac{1}{\left(\left(u_{0}\right)_{\lambda}+w\right)^{\gamma}} .
$$

Now similarly to the proof of Lemma 5.2 , we derive that $w_{\lambda}-w \equiv 0$ in $\Omega^{\prime}$. 
Remark 5.1 In proving (5.11) and (5.12), although there is no Poincaré's inequality on the narrow domain in $\mathbb{H}^{n}$, Sobolev's inequality (2.7) and the fact that $\left|\Omega^{\prime}\right|$ is small helps us to derive (5.12).

Lemma 5.5 If $\partial \Omega$ satisfies the Wiener criterion and $u \in H_{0}^{1}(\Omega) \cap C(\bar{\Omega})(\gamma \leq 1)$ or $u \in$ $H_{\mathrm{loc}}^{1}(\Omega) \cap C(\bar{\Omega})(\gamma>1)$ is a cylindrical solution of problem (1.1), then, for any $\lambda_{0}<\lambda<\lambda_{1}$, we have

$$
w(\xi)<w_{\lambda}(\xi)
$$

for $\xi \in \Sigma_{\lambda}$ with respect to $t$.

Proof Step 1. If $\lambda$ is sufficiently close to $\lambda_{0}$, we have $w>0$ in $\Sigma_{\lambda}$ by Lemma 5.2 and $w \leq w_{\lambda}$ on $\partial \Sigma_{\lambda}$. Since $w=w_{\lambda}$ on the hyperplane $T_{\lambda}$, we get that $w \leq w_{\lambda}$ in $\Sigma_{\lambda}$, and then $w<w_{\lambda}$ on $\Sigma_{\lambda}$ by Lemma 5.4 .

Step 2. Since Step 1 provides a starting point, we can move the plane $T_{\lambda}$ to the right keeping $w \leq w_{\lambda}$ on $\Sigma_{\lambda}$ to its limiting position $\lambda_{1}$. We let

$$
\bar{\lambda}=\sup \left\{\lambda \mid w \leq w_{\mu} \text { in } \Sigma_{\mu}, \mu \leq \lambda\right\}
$$

and claim that $\bar{\lambda}=\lambda_{1}$. Indeed, suppose $\bar{\lambda}<\lambda_{1}$; we will show that $T_{\lambda}$ can further be moved to the right, that is, there exists $\varepsilon>0$ such that, for any $\lambda \in(\bar{\lambda}, \bar{\lambda}+\varepsilon)$,

$$
w \leq w_{\lambda}
$$

This is a contradiction to the definition of $\bar{\lambda}$. Hence $\bar{\lambda}=\lambda_{1}$.

Now we will prove that (5.18) holds on $\Sigma_{\bar{\lambda}+\varepsilon}$. Let us divide $\Sigma_{\bar{\lambda}+\varepsilon}$ into two parts: $\Sigma_{\bar{\lambda}-\varepsilon}$ and $\Sigma_{\bar{\lambda}+\varepsilon} \backslash \Sigma_{\bar{\lambda}-\varepsilon}$, where $\varepsilon>0$ is to be chosen.

Since $w_{\lambda}-w \geq c_{\varepsilon}>0$ in $\Sigma_{\bar{\lambda}-\varepsilon}$ and $w_{\lambda}-w$ depends continuously on $\lambda$, there exists $\varepsilon>0$ sufficiently small such that, for all $\lambda \in(\bar{\lambda}, \bar{\lambda}+\varepsilon)$,

$$
w_{\lambda}-w \geq 0 \quad \text { in } \Sigma_{\bar{\lambda}-\varepsilon}
$$

On the other hand, since $w_{\lambda}-w \geq 0$ on $\partial\left(\Sigma_{\bar{\lambda}+\varepsilon} \backslash \Sigma_{\bar{\lambda}-\varepsilon}\right)$ for $\varepsilon>0$ as before, we have by Lemma 5.4 that

$$
w_{\lambda}-w \geq 0 \quad \text { in } \Sigma_{\bar{\lambda}+\varepsilon} \backslash \Sigma_{\bar{\lambda}-\varepsilon} .
$$

Combining (5.19) and (5.20), there follows (5.18).

Then $w \leq w_{\lambda}$ on $\Sigma_{\lambda}$. Using Lemma 5.4, we obtain $w<w_{\lambda}$ on $\Sigma_{\lambda}$, and now (5.17) is proved.

Proof of Theorem 1.1 Lemma 4.4 and 5.5 imply (1.5).

\section{Conclusions}

In this paper, we obtained the monotonicity of cylindrical solutions to the Dirichlet boundary value problem to singular semilinear subelliptic equation on the Heisenberg group. It is a generalization of the corresponding result in the classical Euclidean setting. 


\section{Acknowledgements}

The authors are grateful to anonymous reviewers for their careful reading of this paper and their insightful comments and suggestions, which improved the paper a lot.

\section{Funding}

This work is supported by the Natural Science Basic Research plan in Shaanxi Province of China (Grant No. 2016JM1023). The first author partially supported by NSFC (Grant No. 11471188) and the National Science Foundation for Young Scientists of China (Grant No. 11601427).

\section{Availability of data and materials}

Not applicable.

\section{Ethics approval and consent to participate}

Not applicable.

\section{Competing interests}

The authors declare that they have no competing interests.

\section{Consent for publication}

Not applicable.

\section{Authors' contributions}

Both authors read and approved the final manuscript.

\section{Publisher's Note}

Springer Nature remains neutral with regard to jurisdictional claims in published maps and institutional affiliations.

\section{Received: 3 August 2017 Accepted: 2 January 2018 Published online: 12 January 2018}

\section{References}

1. Crandall, MG, Rabinowitz, PH, Tartar, L: On a Dirichlet problem with a singular nonlinearity. Commun. Partial Differ. Equ. 2, 193-222 (1977)

2. Lazer, AC, McKenna, PJ: On a singular nonlinear elliptic boundary-value problem. Proc. Am. Math. Soc. 111, 721-730 (1991)

3. Boccardo, L, Orsina, L: Semilinear elliptic equations with singular nonlinearities. Calc. Var. Partial Differ. Equ. 37(3-4), 363-380 (2010)

4. Canino, A, Degiovanni, M: A variational approach to a class of singular semilinear elliptic equations. J. Convex Anal. 11(1), 147-162 (2004)

5. Canino, A, Grandinetti, M, Sciunzi, B: Symmetry of solutions of some semilinear elliptic equations with singular nonlinearities. J. Differ. Equ. 255, 4437-4447 (2013)

6. Birindelli, I, Prajapat, J: Nonlinear Liouville theorems in the Heisenberg group via the moving plane method. Commun. Partial Differ. Equ. 24(9-10), 1875-1890 (1999)

7. Xu, CJ: The Dirichlet problems for a class of semilinear sub-elliptic equations. Nonlinear Anal. 37(8), 1039-1049 (1999)

8. Yuan, Z, Yuan, G: Dirichlet problems for linear and semilinear sub-Laplace equations on Carnot groups. J. Inequal. Appl. 2012, $136(2012)$

9. Jerison, D: Boundary regularity in the Dirichlet problem for $\square_{b}$ on CR manifolds. Commun. Pure Appl. Math. 36(2), 143-181 (1983)

10. Jerison, D: The Dirichlet problem for the Kohn Laplacian on the Heisenberg group, University Microfilms International (1980)

11. Danielli, D, Garofalo, N, Nhieu, DM: Notions of convexity in Carnot groups. Commun. Anal. Geom. 11(2), 263-342 (2003)

12. Montefalcone, F: Some relations among volume, intrinsic perimeter and one-dimensional restrictions of BV functions in Carnot groups. Adv. Math. 159(1), 51-67 (2005)

13. Garofalo, N, Segala, F: Estimates of the fundamental solution and Wiener's criterion for the heat equation on the Heisenberg group. Indiana Univ. Math. J. 39(4), 1155-1196 (1990)

14. Negrini, P, Scornazzani, V: Wiener criterion for a class of degenerate elliptic operators. J. Differ. Equ. 66(2), 151-164 (1987)

15. Chen, W, Li, C: Methods on Nonlinear Elliptic Equations. AIMS Book Series, vol. 4 (2010)

16. Chen, W, Li, C: Classification of solutions of some nonlinear elliptic equations. Duke Math. J. 63(3), 615-622 (1991)

17. Chen, W, Li, C, Ou, B: Classification of solutions for an integral equation. Commun. Pure Appl. Math. 59(3), 330-343 (2006)

18. Gidas, B, Ni, WM, Nirenberg, L: Symmetry and related properties via the maximum principle. Commun. Math. Phys. 68, 209-243 (1979)

19. Bonfiglioli, A, Lanconelli, E, Uguzzoni, F: Stratified Lie Groups and Potential Theory for Their Sub-Laplacians. Springer Monogr. Math. Springer, New York (2007)

20. Bony, JM: Principe du Maximum, inégalité de Harnack et unicité du problème de Cauchy pour les opérateurs elliptiques dégénérés. Ann. Inst. Fourier (Grenoble) 19, 277-304 (1969)

21. Folland, GB: Fundamental solution for subelliptic operators. Bull. Am. Math. Soc. 79, 373-376 (1979)

22. Hörmander, L: Hypoelliptic second order differential equations. Acta Math. 119, 147-171 (1967)

23. Birindelli, I, Cutrì, A: A semi-linear problem for the Heisenberg Laplacian. Rend. Semin. Mat. Univ. Padova 94, 137-153 (1995) 
24. Wu, ZQ, Yin, JX, Wang, CP: Elliptic and Parabolic Equations, pp. 107-109. Springer, Berlin (2006)

25. Garofalo, N, Nhieu, DM: Isoperimetric and Sobolev inequalities for Carnot-Carathéodory spaces and the existence of minimal surfaces. Commun. Pure Appl. Math. 49, 1081-1144 (1996)

26. Lu, GZ: Weighted Poincaré and Sobolev inequalities for vector fields satisfying Hörmander's condition and applications. Rev. Mat. Iberoam. 8(3), 367-439 (1992)

27. Li, P: Geometric Analysis. Cambridge University Press, Cambridge (2012)

28. Ma, L, Wei, JC: Properties of positive solutions to an elliptic equation with negative exponent. J. Funct. Anal. 254(4), 1058-1087 (2008)

29. Tran, KD: Regularity of solutions for quasilinear subelliptic equations on Heisenberg group. Dissertations \& Theses Gradworks (2011)

Submit your manuscript to a SpringerOpen ${ }^{\circ}$ journal and benefit from:

- Convenient online submission

- Rigorous peer review

- Open access: articles freely available online

- High visibility within the field

- Retaining the copyright to your article

Submit your next manuscript at $\gg$ springeropen.com 